\title{
Observation of Chaotic Dynamics in Dilute Sheared Aqueous Solutions of CTAT
}

\author{
Ranjini Bandyopadhyay, Geetha Basappa, and A. K. Sood \\ Department of Physics, Indian Institute of Science, Bangalore 560 012, India
}

\begin{abstract}
The nonlinear flow behavior of a viscoelastic gel formed due to entangled, cylindrical micelles in aqueous solutions of the surfactant cetyl trimethylammonium tosilate (CTAT) has been studied. On subjecting the system to a step shear rate lying above a certain value, the shear and normal stresses show interesting time dependent behavior. The analysis of the measured time series shows the existence of a finite correlation dimension and a positive Lyapunov exponent, unambiguously implying that the dynamics can be described by that of a dynamical system with a strange attractor whose dimension increases with the increase in shear rate.
\end{abstract}

Systems of giant wormlike micelles formed in certain surfactant solutions are known to show very unusual nonlinear rheology. In steady shear, the shear stress $\sigma$ saturates to a constant value while the first normal stress difference increases roughly linearly with shear rate $\dot{\gamma}$ $[1,2]$. The constitutive model of viscoelastic behavior of wormlike micellar systems which incorporates reptation and reaction dynamics (breakage and recombination of micelles) predicts a mechanical instability of the shear banding type [3] where bands supporting high shear rates (low viscosity) coexist with regions of lower shear rates (higher viscosity). Flow birefringence [4] and nuclear magnetic resonance velocity imaging [5] have revealed the existence of banded flow in the shear stress plateau. An alternative explanation for the nonmonotonicity of the flow curve has also been proposed [6,7] in terms of the coexistence of two thermodynamically stable phases, isotropic and nematic, present in the sheared solution. Berret [6] observed damped, periodic oscillations in the stress relaxation of $\mathrm{CPyCl}-\mathrm{NaSal}$ solution at a surfactant volume fraction $\phi$ of $12 \%$ on the application of controlled shear rates $\dot{\gamma}$. Grand et al. [8] have shown the existence of a metastable branch in the flow curve of dilute $\mathrm{CPyCl}-\mathrm{NaSal}$ (cetylpyridinium chloride-sodium salicylate) solution supporting stresses higher than that observed in the stress plateau. They have explained their results in terms of shear banding. Previous observations of shear stress fluctuations in CTAB-NaSal solutions have been explained in terms of shear thickening induced by the growth and retraction of shear induced structures [9].

The rheology of CTAT (cetyl trimethylammonium tosilate) has been examined extensively in the linear viscoelastic regime [10]. Above the Kraft temperature of $23{ }^{\circ} \mathrm{C}$ and at low concentrations $(C<0.04 \mathrm{wt} \%)$, spherical micellar solutions are formed which exhibit Newtonian flow behavior. At higher surfactant concentrations $(0.04<C<$ $0.9 \mathrm{wt} \%$ ), cylindrical wormlike micelles are formed which get entangled at $C>0.9 \mathrm{wt} \%$ to form clear viscoelastic gels [10]. The purpose of this Letter is to report interesting time dependence of the shear and normal stresses after subjecting the system to a step shear rate lying in the plateau region. Our detailed analysis shows unambiguously that the observed dynamics can be described as that of a low dimensional, dynamical system with a strange attractor.

Our experiments were done on dilute aqueous solutions of CTAT, 1.35 wt $\%$ at $25^{\circ} \mathrm{C}$. Following the measurements of the elastic modulus $G^{\prime}(\omega)$ and the viscous modulus $G^{\prime \prime}(\omega)$ in the linear rheology regime and the flow curve ( $\sigma$ vs $\dot{\gamma}$ ) in the constant stress mode, controlled shear rate experiments were performed wherein shear stress and normal stress showed interesting time dependence. The time series obtained for both types of stresses have been analyzed by two methods to extract certain invariant characteristics, metric and dynamical, of the nonlinear dynamics such as correlation dimensions, the embedding dimensions, and Lyapunov exponents. The analysis following the algorithm due to Grassberger and Procaccia [11] shows that there is a finite correlation dimension. Another method due to Gao and Zheng [12] yields an estimate of the positive Lyapunov exponent. This analysis unambiguously shows that the observed time dependence of the signal is not due to stochastic noise, but has its origin in deterministic chaotic dynamics. We note that Noronha et al. have shown the existence of chaotic dynamics in the jerky flow of some metal alloys undergoing plastic deformation [13] which also show a nonmonotonic flow curve. Recently, after the completion of our present studies [14], we became aware of experiments by Soltero et al. [15] on the nonlinear rheology of CTAT at 5 and $10 \mathrm{wt} \%$ which show the existence of a plateau region at $\dot{\gamma}>\left(2 \tau_{R}\right)^{-1}$ and oscillations in the stress and birefringence in $5 \mathrm{wt} \%$ CTAT at $\dot{\gamma}=100 \mathrm{~s}^{-1}$.

The CTAT-water samples used in our experiments were prepared by adding appropriate amounts of CTAT to distilled and deionized water and were allowed to equilibrate for at least one week. For our experiments we used a stress controlled rheometer with temperature control and software for shear rate control (Rheolyst AR-1000N, T.A. Instrument, U.K.). The rheometer was also equipped with eight strain gauge transducers capable of measuring the normal force to an accuracy of $10^{-4} \mathrm{~N}$. The measurements were made using a cone-and-plate geometry of 
cone diameter $4 \mathrm{~cm}$ and angle $1^{\circ} 59^{\prime \prime}$. All the experiments reported here have been done on fresh samples from the same batch to eliminate sample history effects. The frequency response measurements of CTAT $1.35 \mathrm{wt} \%$ by subjecting it to small oscillatory stresses in the linear regime reveal a very poor fit to the Maxwell model, as seen in insets (a) and (b) of Fig. 1, in contrast to the behavior at higher CTAT concentrations [10]. The reason for the failure of the Maxwell model can be that the two relaxation times $\tau_{b}$ and $\tau_{\text {rep }}$ are not widely different $\left(\tau_{b} \sim 1.79 \mathrm{~s}\right.$ as estimated from the Cole-Cole plot and $\tau_{\text {rep }} \sim 5 \mathrm{~s}$ obtained from the crossover frequency $\omega_{\mathrm{CO}}=0.3 \mathrm{rad} \mathrm{s}^{-1}$ in the $G^{\prime}$ and $G^{\prime \prime}$ plots, which gives an approximate estimate of the relaxation time $\tau_{R} \sim 3 \mathrm{~s}$ ). We recall that in the Maxwell model, $G(t)=G_{0} e^{-t / t_{R}}$, where $\tau_{R}=\left(\tau_{b} \tau_{\text {rep }}\right)^{1 / 2}, \tau_{b}$ and $\tau_{\text {rep }}$ are the breaking and reptation times of the micelles with $\tau_{b} \ll \tau_{\text {rep }}$ [16].

Figure 1 shows the metastable flow curve measured under conditions of controlled stress (time elapsed between acquisition of successive data points $=2 \mathrm{~s}$ ) for the CTAT solution of concentration $1.35 \%$ by weight. Interestingly, even though CTAT 1.35 wt \% is not strictly Maxwellian, its flow curve shows a smooth transition to a plateau region above a critical shear rate similar to the ones observed in surfactant solutions of $\mathrm{CPyCl} / \mathrm{NaSal}$ [6-8] and concentrated CTAT [15], which show Maxwellian behavior in $G(\omega)$. For $10<\dot{\gamma}<200 \mathrm{~s}^{-1}, \sigma \sim \dot{\gamma}^{\alpha}$ where $\alpha=0.06 \pm 0.004$, implying a very weak concentration dependence [17]. This value of $\alpha$ is very much smaller than that observed in the flow curve of the $\mathrm{CPyCl} /$ hexanol $/ \mathrm{NaCl} /$ water system $(\alpha=0.3)$ which undergoes an isotropic-nematic transition at $\phi=31 \%$ [17]. This observation indicates that the plateau in the flow curve of our system is due to shear banding rather than the coexistence of the isotropic and nematic phases

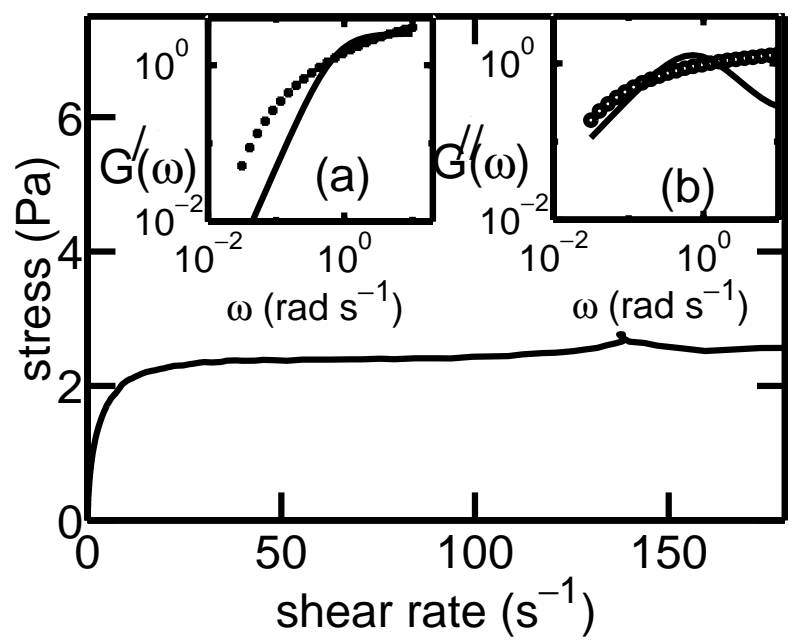

FIG. 1. Metastable flow curve of CTAT $1.35 \mathrm{wt} \%$ at $25{ }^{\circ} \mathrm{C}$, measured under conditions of controlled stress. Insets (a) and (b) show $G^{\prime}(\omega)$ (shown by squares) and $G^{\prime \prime}(\omega)$ (shown by circles). The solid lines show the fits to the Maxwell model.
[17]. We now discuss our stress relaxation measurements on imposing step shear rates of different magnitudes to the samples. For low shear rates, $\dot{\gamma} \leq 22 \mathrm{~s}^{-1}$, the stress relaxes monotonically to a steady state value in a few seconds. For higher shear rates, the stress initially relaxes $(t<100 \mathrm{~s})$ and then oscillates in time, as shown in Figs. 2(a)-2(e) for a few typical values of applied shear rates. Figure 2(f) shows the oscillations in the normal stress measured along with the shear stress (curve c) at $\dot{\gamma}=100 \mathrm{~s}^{-1}$. On raising the temperature, the oscillations in the stress relaxation are found to decrease in amplitude and disappear completely at a temperature of $35^{\circ} \mathrm{C}$ [Fig. 2(g)]. This may be because of a decrease in the width of the stress plateau in the flow curve with increasing temperatures [7]. The power spectra calculated from the Fourier transforms of the stress autocorrelation functions have been computed from the time-dependent stress data as shown in Fig. 3. The initial decay of the observed stress $(t<100 \mathrm{~s})$ has not been included in the calculations of the power spectrum. The power spectra reveal that for $\dot{\gamma}=22.5 \mathrm{~s}^{-1}$, there is only one peak at $0.009 \mathrm{~s}^{-1}$ corresponding to a time scale of $110 \mathrm{~s}$. At $\dot{\gamma}=75 \mathrm{~s}^{-1}$, the power spectrum exhibits two dominant peaks at 0.01 and $0.015 \mathrm{~s}^{-1}$, corresponding to time scales of 100 and $67 \mathrm{~s}$. At higher shear rates, there are

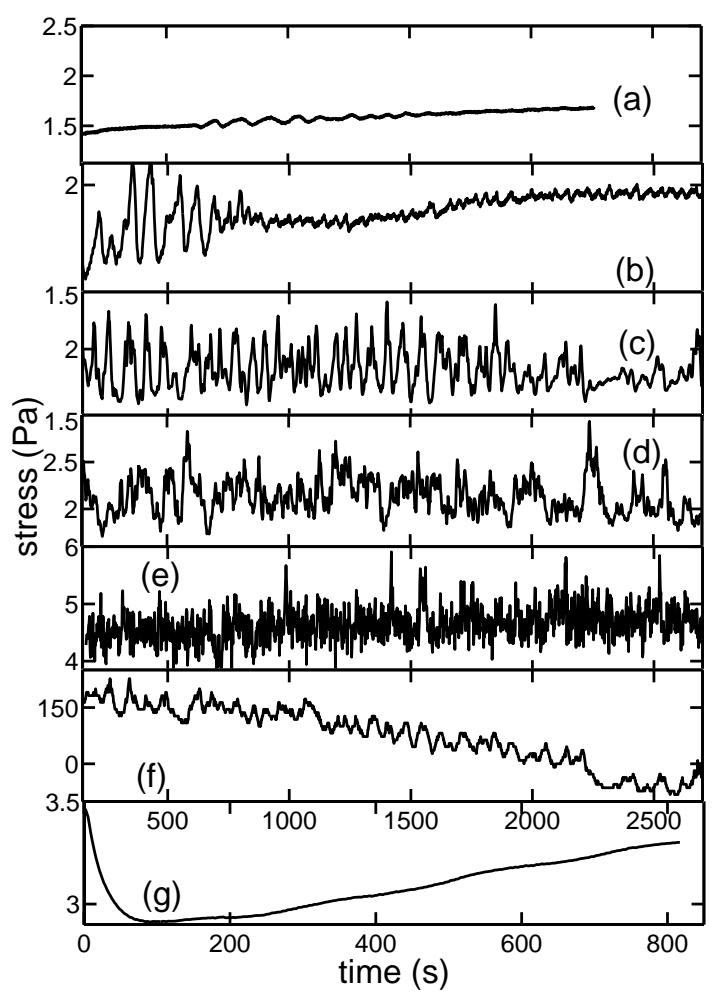

FIG. 2. Shear stress relaxation in CTAT $1.35 \%$ on subjecting the sample to step shear rates of (a) $22.5 \mathrm{~s}^{-1}$, (b) $75 \mathrm{~s}^{-1}$, (c) $100 \mathrm{~s}^{-1}$, (d) $138 \mathrm{~s}^{-1}$, (e) $175 \mathrm{~s}^{-1}$ at $25^{\circ} \mathrm{C}$. (f) shows the time-dependent decay of the normal stress on application of $\dot{\gamma}=100 \mathrm{~s}^{-1}$, also at $25^{\circ} \mathrm{C}$. Figure $2(\mathrm{~g})$ shows the disappearance of the time-dependent oscillations at $35^{\circ} \mathrm{C}$ at $\dot{\gamma}=100 \mathrm{~s}^{-1}$. 


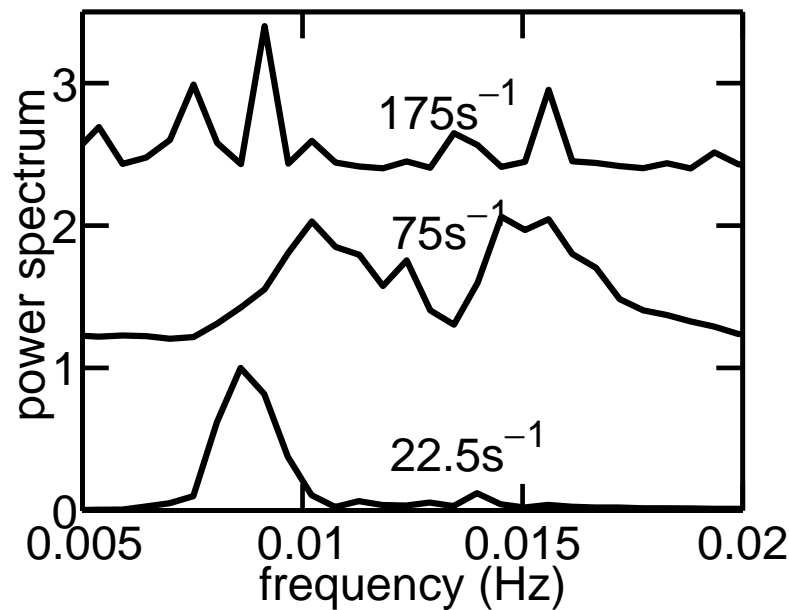

FIG. 3. Power spectra of the stress relaxation data at $\dot{\gamma}=22.5$, 75 , and $1.75 \mathrm{~s}^{-1}$.

many more frequency components. These time scales inferred from Fig. 3 may be understood as being due to the realignment of macrodomains which form as a result of shear banding. Stick-slip between these domains due to the application of shear can result in the observed time-dependent behavior.

We will now present the analysis of the data to look for any pattern expected in a deterministic dynamical system which would distinguish it from stochastic noise. There is a short time predictability in deterministic chaos as compared to no predictability at all in stochastic noise. Let $\sigma_{j}=\sigma(j \Delta t)$ denote the time series shown in Fig. 2, consisting of stresses measured at regular time intervals $\Delta t(=1.8 \mathrm{~s})$, with $j=1$ to $N(N=1500)$. The time series is used to construct an $m$-dimensional vector $\vec{X}_{i}=$ $\left(\sigma_{i}, \sigma_{i+L}, \ldots, \sigma_{i+(m-1) L}\right)$ where $m$ is the embedding dimension and $L$ is the delay time. The embedding theorem of Takens ensures that the dynamics of the original system is represented by $F: \vec{X}_{i} \rightarrow \vec{X}_{i+1}$, provided that the embedding dimension $m$ and the delay time $L$ are properly chosen [18]. A useful way to characterize the dynamical system is by the correlation dimension $\nu$ [11] of the (strange) attractor towards which the phase space trajectories converge in the asymptotic limit. The correlation integral $C(R)$ is defined in an $m$-dimensional phase space as $C(R)=$ $\lim _{N \rightarrow \infty} \frac{1}{N^{2}} \sum_{i, j=1}^{N} H\left(R-\left|\vec{X}_{i}-\vec{X}_{j}\right|\right)$, where $H(x)$ is the Heaviside functions and $\left|\vec{X}_{i}-\vec{X}_{j}\right|$ is the distance between the pair of points $(i, j)$ in the $m$-dimensional embedding space. The sum in the expression for $C(R)$ gives the number of point pairs separated by a distance less than $R$. For small $R$ 's, $C(R)$ is known to scale as $C(R) \sim R^{\nu}$, where the correlation dimension $\nu$ gives us useful information about the local structure of the attractor [11]. The exponent $\nu$ is obtained as a function of $\log (R)$ from the plot of $\log [C(R)]$ versus $\log (R)$ (Fig. 4). A plateau in the plot of $\nu$ versus $\log (R)$ gives the correct $\nu$ for a chosen embedding dimension $m$. If the attractor is unfolded by choosing a large enough $m$, then the correlation dimension $\nu$ becomes independent of the value of the embedding dimension $m$. The value of $m$ at which this independence sets in is the correct embedding dimension, and the corresponding $\nu$ is the correlation dimension. If an experimental signal satisfies $\nu<m$, then the signal is due to deterministic chaos rather than random noise [11].

Figure 4(a) shows the typical calculations of the correlation dimension $\nu$ of the attractor to which the stress trajectories asymptotically converge for a shear rate of $100 \mathrm{~s}^{-1}$. This gives $m=4$ and $\nu=2.8$. Figure 4(b) reveals a monotonically increasing behavior of $\nu$ with $\dot{\gamma}$. We see that $\nu \sim 1$ for stress relaxation data at $\dot{\gamma}=22.5 \mathrm{~s}^{-1}$ where the power spectrum [Fig. 3] shows a single frequency. This is indeed expected as $\nu=1$ for a singly periodic motion (limit cycle), $\nu=2$ for a biperiodic torus and $\nu>2$ for a strange attractor [18]. We see that the correlation dimension $\nu>2$ above a shear rate of $75 \mathrm{~s}^{-1}$. The dynamics of the stress relaxation thus appears to take place on the surfaces of attractors of fractal dimensions that increase with the increase in the applied shear rate. The procedure has been repeated for different data sets acquired at different times and the values of $\nu$ calculated in these cases are found to agree to within $15 \%$. We have also calculated the correlation dimensions of the attractors on which the normal stress orbits lie when CTAT $1.35 \%$ was subjected to high shear rates. The normal stress is also found to show low-dimensional, deterministic dynamics [19]

The existence of a positive Lyapunov exponent is the most reliable signature of chaotic dynamics [18]. The Lyapunov exponent characterizes how the distance between two neighboring points in phase space evolves in time. Defining $d_{i j}(k)=\left\|\vec{X}_{i+k}-\vec{X}_{j+k}\right\|$, the Euclidean
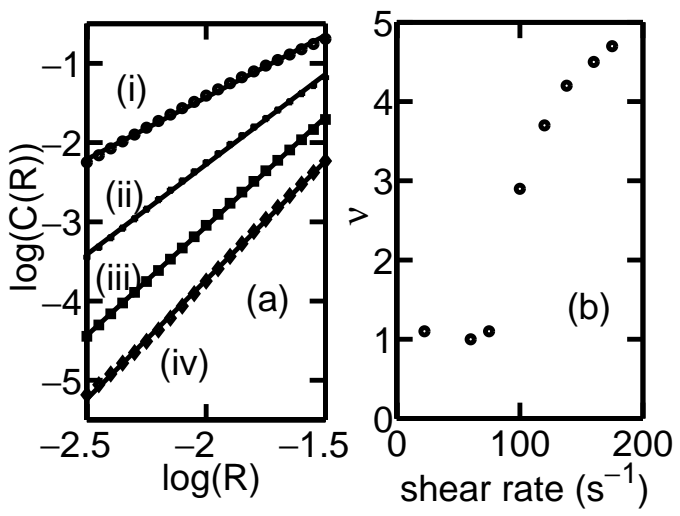

FIG. 4. (a) shows the plot of $\log [C(R)]$ vs $\log (R)$ of the stress trajectories at $\dot{\gamma}=100 \mathrm{~s}^{-1}$ for $m=2$ to 5 [(i)-(iv)]. The slopes of the plots give the following values of $\nu$ : (i): $\nu=$ 1.6 for $m=2$; (ii): $\nu=2.3$ for $m=3$; (iii): $\nu=2.8$ for $m=4$; and (iv): $\nu=2.8$ for $m=5$. (b) shows the correlation dimensions calculated as a function of shear rate $\dot{\gamma}$. 


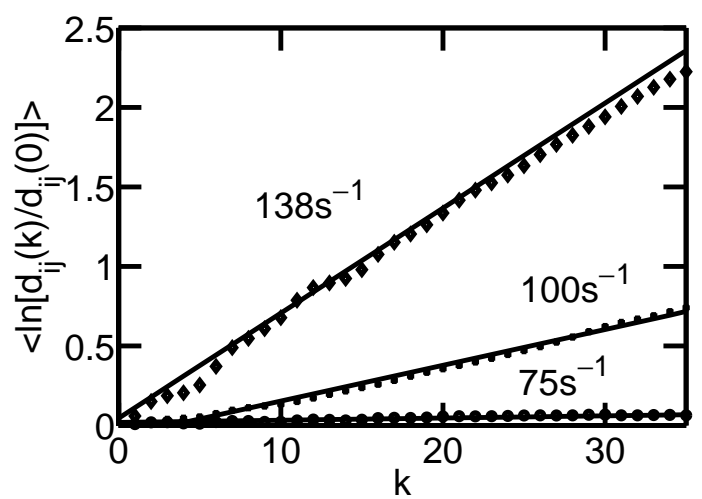

FIG. 5. Calculation of the Lyapunov exponent for $\dot{\gamma}=75,100$ and $138 \mathrm{~s}^{-1}$. The Lyapunov exponent which may be estimated from the slope of the curves is $\sim 0$ at $\dot{\gamma}=75 \mathrm{~s}^{-1}$ and becomes positive at higher shear rates.

distance between two vectors constructed using the embedding theorem by $k$ iterations of the dynamics $F$, and plotting $\left\langle\ln \left[d_{i j}(k) / d_{i j}(0)\right]\right\rangle$ as a function of $k$, we calculate the Lyapunov exponent by using the relation $\lambda=S / \Delta t \ln (2)$, where $S$ is the slope of the plot. It is seen that $\lambda$ becomes positive at shear rates $>75 \mathrm{~s}^{-1}$, indicating the onset of chaos at these shear rates (Fig. 5).

In order to quantitatively understand our results, we need to set up space and time-dependent, nonlinear, coupled differential equations in at least four phase space variables. These equations will be the equations of motion, for example, the continuity and momentum balance equations together with the constitutive relation between viscoelastic stress and shear rate. The starting point can be the JohnsonSegalman (JS) model [20] proposed in the context of "spurt effect" of polymers which has generically similar nonmonotonic behavior to the reptation-reaction model of wormlike micelles. In the JS model, the viscoelastic nature of the polymer is accounted for by writing the total stress as the sum of a Newtonian part and a deformation history dependent viscoelastic part. This model predicts damped oscillations in the stress in controlled shear rate conditions [6,19]. We believe that the coupling of the mean micellar length to the shear rate [21], the dynamics of the mechanical interfaces [22] and the flowconcentration coupling [23] should be incorporated in the JS model. A model constructed by incorporating these additional features, which takes into account the nonlinear coupling between the relevant dynamical variables like shear and normal stresses, shear rate, concentration profiles, and micellar length distributions is most likely to exhibit the chaotic behavior that we observe.

In summary, we have proved the existence of chaotic dynamics in the rheology of dilute, aqueous solutions of
CTAT. Our analysis unambiguously shows that the minimum embedding dimension required to describe the shear banding instability is four. Our experiments and analyses will favor the explanation of the nonmonotonic flow behavior in terms of mechanical instability rather than the coexistence of isotropic and nematic phases in the sheared solution. The presence of the nematic phase for a very low concentration regime as in our experiments is highly unlikely. We hope that our experiments will motivate full theoretical modeling of the shear banding phenomenon.

We thank S. Ramaswamy, V. Kumaran, and P. R. Nott for the use of the rheometer. R. B. thanks the CSIR and AKS thanks BRNS, India for financial support.

[1] H. Rehage and H. Hoffmann, Mol. Phys. 74, 933 (1991).

[2] N. A. Spenley, M.E. Cates, and T.C.B. McLeish, Phys. Rev. Lett. 71, 939 (1993).

[3] N. A. Spenley, X. F. Yuan, and M.E. Cates, J. Phys. II (France) 6, 551 (1996).

[4] R. Makhloufi et al., Europhys. Lett. 32, 253 (1995).

[5] R. W. Mair and P. T. Callaghan, Europhys. Lett. 36, 719 (1996).

[6] J. F. Berret, D. C. Roux, and G. Porte, J. Phys. II (France) 4, 1261 (1994); J. F. Berret, Langmuir 13, 2227 (1997).

[7] G. Porte, J. F. Berret, and J. L. Harden, J. Phys. II (France) 7, 459 (1997).

[8] C. Grand, J. Arrault, and M. E. Cates, J. Phys. II (France) 7, 1071 (1997).

[9] C. Liu and D. J. Pine, Phys. Rev. Lett. 77, 2121 (1996).

[10] J.F.A. Soltero et al., Langmuir 11, 3337 (1995); J.F. A. Soltero, J.E. Puig, and O. Manero, Langmuir 12, 2654 (1996).

[11] P. Grassberger and I. Procaccia, Phys. Rev. Lett. 50, 346 (1983).

[12] J. Gao and Z. Zheng, Phys. Rev. E 49, 3807 (1994).

[13] S. J. Noronha et al., Int. J. Bifurcation Chaos Appl. Sci. Eng. 7, 2577 (1997).

[14] A. K. Sood, R. Bandyopadhyay, and G. Basappa, Pramana J. Phys. 53, 223 (1999).

[15] J. F. A. Soltero et al., Langmuir 15, 1604 (1999).

[16] M. E. Cates, Macromolecules 20, 2289 (1987).

[17] J.F. Berret et al., Europhys. Lett. 25, 521 (1994); P. D. Olmsted and C. Y.D. Lu, Faraday Discuss. Chem. Soc. 112, 183 (1999).

[18] E. Ott, in Chaos in Dynamical Systems (Cambridge University Press, Cambridge, England, 1993).

[19] R. Bandyopadhyay and A. K. Sood (unpublished).

[20] M. Johnson and D. Segalman, J. Non-Newton. Fluid Mech. 2, 255 (1977).

[21] M.E. Cates and M.S. Turner, Europhys. Lett. 11, 681 (1990).

[22] X.F. Yuan, Europhys. Lett. 46, 542 (1999).

[23] V. Schmitt, C. M. Marques, and F. Lequeux, Phys. Rev. E 52, 4009 (1995). 